\title{
Plataformas y Herramientas Educativas como parte del PLE del Docente. Caso Asistente Digital para Planeación Curricular ConTIC
}

\section{Platforms and Educational Tools as part of PLE of Teacher. Case for Planning Digital Assistant Curriculum ConTIC}

\author{
DOI: http://dx.doi.org/10.17981/ingecuc.12.1.2016.11
}

Artículo de Investigación Científica - Fecha de Recepción: 30 de Septiembre de 2015 - Fecha de Aceptación: 14 de Diciembre de 2015

\section{Mónica María Rojas Rincón}

Magister en Ingeniería de Sistemas y Computación. Politécnico Colombiano Jaime Isaza Cadavid. Medellín (Colombia) mmrojas@elpoli.edu.co

\author{
Gustavo Alberto Moreno López \\ Magister en Ingeniería. Politécnico Colombiano Jaime Isaza Cadavid. Medellín (Colombia) \\ gamoreno@elpoli.edu.co
}

Claudia Alejandra Rosero Noguera

Magister en Ingeniería. Politécnico Colombiano Jaime Isaza Cadavid. Medellín (Colombia) carosero@elpoli.edu.co

Para citar este artículo / To reference this paper:

M. M. Rojas Rincón, G. A. Moreno López y C. A. Rosero Noguera, "Plataformas y herramientas educativas como parte del PLE del docente. Caso asistente digital para planeación curricular ConTIC", INGE CUC, vol. 12, no. 1, pp. 99-106, 2016. DOI: http://dx.doi.org/10.17981/ingecuc.12.1.2016.11

\begin{abstract}
Resumen-- Las TIC tienen un papel importante en la educación. Enmarcado en los entornos de aprendizaje personal -PLE, donde cada individuo identifica los recursos que emplea para aprender, crear y compartir, el docente como guía, tiene un reto de apropiarse también de las TIC. Esta presentación hace parte del proyecto "Modelo de asistente para la construcción pedagógica de microcurrículos apoyado en TIC para instituciones educativas de básica y media”, bajo el programa "Apropiación pedagógica de las TIC en las escuelas innovadoras del CIER Occidente", apoyado por Colciencias-MEN. Para el proyecto se definió la metodología de investigación basada en diseño (IBD). En este documento se indica un resumen de las herramientas y plataformas educativas, se presenta una herramienta digital que actúa como asistente para la planeación curricular denominado conTIC, y una propuesta general del entorno de aprendizaje personal -PLE del docente que incluye a conTIC.
\end{abstract}

Palabras claves-- TIC; plataformas; herramientas; PLE; Docente; Asistente para planear la clase.

\begin{abstract}
ICT has an important role in education. Framed in personal learning environments-PLE, where every individual identifies the resources used to learn, create and share, the teacher as how guide, also has a challenge of appropriate ICT. This presentation is part of the Project "Wizard Model for the pedagogical construction of micro curricula supported by ICT, for educational institutions in primary and secondary" under the program "pedagogical appropriation of ICT in innovative schools CIER West", supported by Colciencias-MEN. For the project was defined the methodology, design-based research. This document is a summary of the tools and learning platforms indicated, is presented a digital tool that acts as a wizard for curriculum planning called CONTIC, and a general proposal of teacher PLE which includes conTIC.
\end{abstract}

Keywords-- ICT; Platforms; Tools; PLE; Teacher; Wizard to Plan Class. 


\section{INTRODUCCIÓN}

Sin lugar a dudas las TIC (Tecnologías de la información y la comunicación), han jugado un papel importante en diferentes sectores de la sociedad, y en el sector educativo para favorecer, enriquecer el proceso de enseñanza y aprendizaje.

Las plataformas tecnológicas de e-learning como lo definen Carneiro y otros [1] p.99 "son programas de ordenador que se utilizan para la creación, gestión y distribución de actividades formativas a través de la web. Estas aplicaciones facilitan entornos de enseñanza-aprendizaje mediante la integración de materiales didácticos, herramientas de comunicación, colaboración y gestión educativa".

En este documento se pretende bajo el contexto de los entornos personales de aprendizaje (PLE), presentar en forma global los recursos TIC que pueden beneficiar al docente, enfatizando en una propuesta de herramienta que actúa como asistente digital de gestión de unidades de aprendizaje denominada conTIC, y la inclusión de esta al diseño del PLE docente.

La propuesta hace parte del proyecto "Modelo de asistente para la construcción pedagógica de microcurrículos apoyado en TIC para instituciones educativas de básica y media", bajo el programa "Apropiación pedagógica de las TIC en las escuelas innovadoras del CIER Occidente", apoyado por Colciencias-MEN. Para efectos de retroalimentación, pruebas y validaciones se aplicó en las Instituciones educativas INEM José Félix de Restrepo y Gabriel García Márquez (GGM) de Medellín.

El problema o necesidad que se planteó en el proyecto, es sobre la integración de las TIC al quehacer diario de los docentes, y que se carece de recursos tecnológicos enfocados a facilitar y asistir a los docentes en su proceso de planeación, organización, implementación y evaluación del currículo. De acuerdo al programa todos a aprender (PTA) del Ministerio de Educación Nacional, se hace necesario fortalecer la planeación de las clases, para aprovechar el espacio y mejorar los resultados de aprendizaje [2]. Además los docentes encuestados en las I.E, INEM y GGM, manifestaron que cuando planean usan un formato en Word o Excel, y que les gustaría tener una herramienta web.

En el resto del artículo está organizado de la siguiente forma, en primer lugar la revisión del contexto de las plataformas genéricas y específicas, el concepto de los PLE, ejemplos de plataformas y herramientas educativas; Luego en resultados, se presenta el asistente curricular digital Contic, la evaluación y la propuesta de un escenario del PLE docente que incluye a conTIC.

\section{REvisión CONCEPTUAL}

Las plataformas educativas se distinguen entre las que son de carácter general y las específicas. Las plataformas específicas según Ana Fernández, tienen el objetivo de:

mejorar la eficacia y eficiencia académica (para una mejor y más rápida enseñanza y aprendizaje), especializándose en determinadas áreas de conocimiento o completando la funcionalidad de las plataformas genéricas. Así se encuentran plataformas especializadas en a) un dominio (competencia o materia) concretas; b) un modelo y/o metodología de aprendizaje específico, o finalmente, c) una tarea específica. Estas plataformas construyen y gestionan los EA siguiendo unos criterios específicos del dominio. [3] p.7

La figura 1 ilustra una clasificación general de las plataformas de apoyo al proceso educativo. Además se encuentran otras herramientas que apoyan la gestión y preparación del proceso educativo.

La siguiente tabla presenta un comparativo general de estas plataformas.

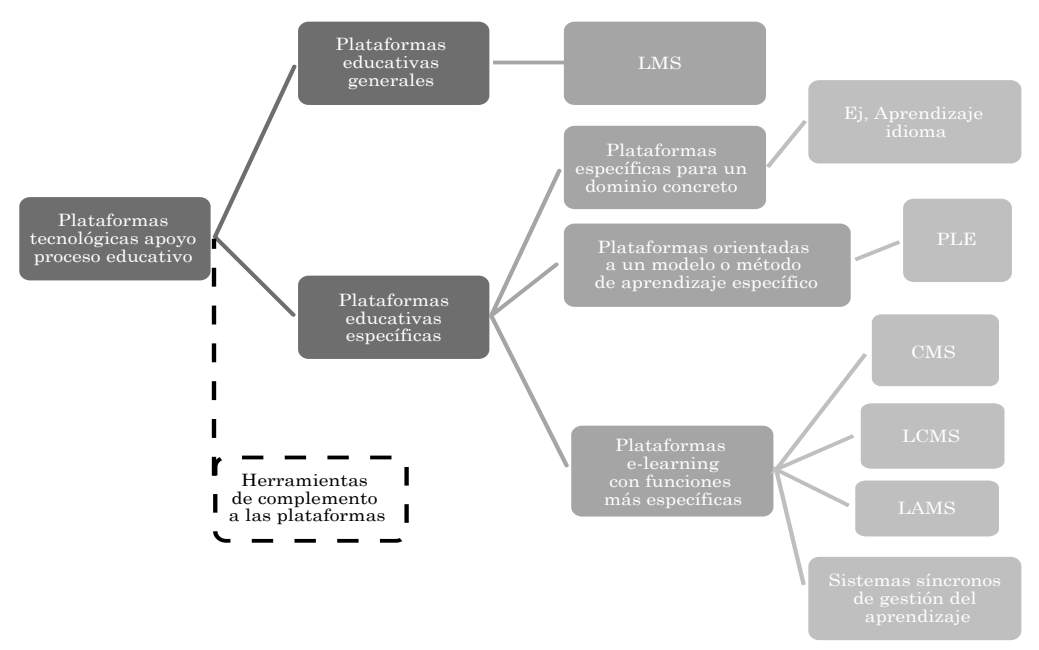

Fig. 1. Clasificación general de plataformas educativas. Fuente: Autor 
TABla I. Comparativo gLOBAL PLATAFormas.

\begin{tabular}{|l|l|l|l|l|}
\hline Características & \multicolumn{1}{|c|}{ LMS } & \multicolumn{1}{c|}{ CMS } & \multicolumn{1}{c|}{ LCMS } & \multicolumn{1}{c|}{ LAMS } \\
\hline Significado & $\begin{array}{l}\text { Sistema de gestión de } \\
\text { aprendizaje }\end{array}$ & $\begin{array}{l}\text { Sistema de gestión } \\
\text { de contenidos }\end{array}$ & $\begin{array}{l}\text { Sistema de gestión de } \\
\text { contenidos de aprendizaje }\end{array}$ & $\begin{array}{l}\text { Sistema de gestión de } \\
\text { actividades de aprendizaje }\end{array}$ \\
\hline Principal función & $\begin{array}{l}\text { Administrar usuarios, } \\
\text { distribuir contenidos y dar } \\
\text { seguimiento del aprendizaje }\end{array}$ & $\begin{array}{l}\text { Administrar y } \\
\text { crear contenidos }\end{array}$ & $\begin{array}{l}\text { Administrar usuarios, } \\
\text { seguimiento aprendizaje, } \\
\text { y crear contenidos }\end{array}$ & $\begin{array}{l}\text { Administrar secuencias de } \\
\text { actividades de aprendizaje }\end{array}$ \\
\hline Integración & & LMS + CMS & Puede integrarse ej con LMS \\
\hline $\begin{array}{l}\text { Soporta } \\
\text { estándares }\end{array}$ & Si varios & Algunos & Si varios & $\begin{array}{l}\text { Si, combinado por ejemplo } \\
\text { con LMS }\end{array}$ \\
\hline
\end{tabular}

Fuente: Autor

En la sesión B, se indican ejemplos de cada una de estas plataformas.

\section{A. Entorno Personal de Aprendizaje - PLE}

De acuerdo a Pampillón [3] p.8 los PLE "no han sido concebidas estrictamente como plataformas educativas”. Pero un PLE contempla a la vez, un enfoque pedagógico como tecnológico.

En Castañeda se define PLE (Personal Learning Environment), o entorno de aprendizaje personal como:

"el conjunto de herramientas, fuentes de información, conexiones y actividades que cada persona utiliza de forma asidua para aprender" [4] p. 23. Es decir, el PLE de las personas se configura por los procesos, experiencias y estrategias que el aprendiz puede y debe poner en marcha para aprender y, en las actuales condiciones sociales y culturales, está determinado por las posibilidades que las tecnologías abren y potencian. [4] p.17

Castañeda comenta que las partes del PLE son:

1. Herramientas y estrategias de lectura: las fuentes de información a las que accedo que me ofrecen dicha información en forma de objeto o artefacto (mediatecas);

2. Herramientas y estrategias de reflexión: los entornos o servicios en los que puedo transformar la información (sitios donde escribo, comento, analizo, recreo, publico), y

3. Herramientas y estrategias de relación: entornos donde me relaciono con otras personas de/con las que aprendo". [4] p.17

Luego en el PLE se integran 3 partes principales, como indica la figura 2:

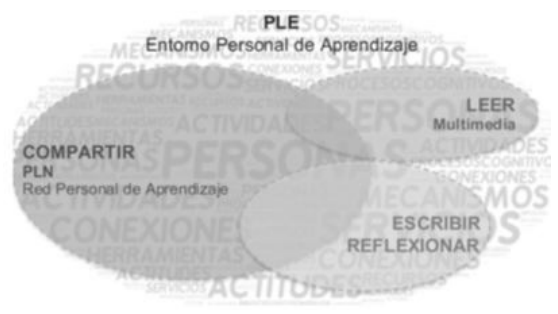

Fig 2. Partes principales del PLE. Fuente: [4]
Como se indica en la figura anterior, y lo resalta Castañeda "no sólo se habla de un PLE individual y formado por mí y "mis cosas" para aprender, sino que se incluye mi entorno social para aprender (Red Personal de Aprendizaje) o PLN", "con sus fuentes y relaciones como parte fundamental de ese entorno" [4] p.19

El enfoque pedagógico del PLE. En la era de la digitalización, las tecnologías, las diferentes posibilidades de conexiones, el aprendizaje está en todas partes. Una de las teorías en la que se sustenta la utilización de las TIC (tecnologías de la información y la comunicación) para el aprendizaje, es el conectivismo, una teoría de aprendizaje para la era digital [5]. EL PLE además se complementa en el "modelo de aprendizaje socio-constructivista en el que el estudiante es el protagonista de su propio aprendizaje, cooperando y colaborando con el grupo para construir nuevos conocimientos" Pampillón [3] p.8.

Varios autores han contemplado el tema de los PLE, como Castañeda [4] p.164, Llamas Nistal y otros [6], Mohd y Shahbodin [7], Moccozet y otros [8], la Universidad de Aveiro [9] con el Sapo Campus, el proyecto Dipro 2.0 [10], el cual está enfocado al "Diseño, producción y evaluación de un entorno de aprendizaje 2.0, para la capacitación del profesorado universitario en la utilización de las Tecnologías de la Información y Comunicación (TIC)”, financiado por el Ministerio de Ciencia e Innovación del Gobierno de España. Hasta ahora en la literatura no se contempla una herramienta de asistente de planeación curricular que sea parte del PLE docente, y aún hace falta más investigación y experiencias con los diseños de PLE. Específicamente en Colombia, sólo se encontró una herramienta relacionada con la planeación del currículo, Currículo Interactivo 2.0 de Eduteka [11], la cual es para currículos de informática.

\section{B. Plataformas educativas}

Dentro de las plataformas educativas generales, tenemos los LMS (Learning Management Systems), sistemas de gestión de aprendizaje, "que se emplea para crear, aprobar, administrar, almace- 
nar, distribuir y gestionar las actividades de formación virtual (puede utilizarse como complemento de clases presenciales o para el aprendizaje a distancia) [12] p.28.

Son ejemplos de LMS libres (Moodle, .LRN, Atutor, Sakai, Claroline, entre otros). Ejemplos de LMS comerciales son (Blackboard, Desire2learn, Firstclass, E-college, entre otros). Otros ejemplos son los empleados para los cursos online abiertos masivos, conocidos como MOOC (Massive Open Online Course), entre ellos Udacity, Coursera, edX, Edmodo, entre otros.

Dentro de las plataformas educativas específicas tenemos, los CMS (Content Management System), sistemas de gestión de contenido, "aplicaciones que permiten la creación, almacenamiento indexado, clasificación, publicación y gestión multiusuario y concurrente del ciclo de vida de los contenidos". Mejoran las capacidades de los LMS, "limitadas al almacenamiento en directorios y a la publicación" [3] p.9. Son ejemplo de estas plataformas, Phone, Silva, Drupal, Wordpress, Joomla, entre otras.

Un LCMS (Learning Content Management System), son sistemas de gestión de aprendizaje y contenidos. "Es un sistema independiente o integrado con el LMS, que incorpora la creación y administración de contenidos de aprendizaje a diferentes niveles para personalizar los recursos a cada alumno" [1] p.103. Entonces un LCMS como indica Ninoriya [13], combina la robustez del CMS integrándolo en el LMS. Algunos ejemplos plataformas LCMS son, Acontent, Xyleme, Open Authoring, Exact.

Un LAMS (Learning Activities Management Systems), son sistemas de gestión de secuencias de actividades de aprendizaje. Como lo indica Pampillón [3] p.10, el LAMS "Tienen como objetivo la construcción de EA instruccionales. LAMS se puede utilizar como un sistema independiente o en complementado con otros sistemas de gestión de aprendizaje (LMS) como Moodle, o .LRN. Ejemplos de estas plataformas son el de la fundación LAMS [14], o CopperCore [15].

Otras plataformas complementarias y que son para tiempo real son EVO, BigBlueButton, Adobe Connect Blackboard Collaborate, Elluminate Live, entre otras.

\section{Herramientas complementarias}

Existen infinidad de recursos como los resalta la Red Latinoamericana de portales educativos RELPE [16], para crear, editar, interactuar, comunicar, compartir, entre otros. Son ejemplos los siguientes:

- Edición de imágenes (Picasa, Gimp, Flickr, twitpic, entre otras).

- Edición de texto (Google Docs, Openoffice, ZohoWriter, entre otras).
- Edición de audio (Mybytes, audacity, sibelius, entre otras).

- Edición video y publicación (Camtasia, Youtube, Vimeo, atubecatcher, Windows movie maker, entre otras).

- Para videoconferencias (Skype, VyEW, SwowDocument, entre otras).

- Para presentaciones (Prezi, Zoho Show, 280Slides, Slideshare).

- Trabajar en la nube (Dropbox, Beluga, Google Apps, entre otras).

- Publicar documentos (Scribd, Cuadernia, issuu, entre otras).

- Realizar mapas mentales y/o conceptuales (Cmaptools, Freemind, mindmeister, Xmind, Spiderscribe, entre otras).

- Usar Wiki (Wikispaces, Wikia, Wikipedia, entre otras).

- Realizar Blogs (Blogger, Wordpress, Posterous, entre otras).

- Usar redes sociales (Twitter, Google +, Facebook, Ning, Linkedim, entre otras).

- Usar mapas (Google maps, Google earth, Umapper, Xpeditions Atlas, Animaps, entre otras).

- Generar comics y dibujos animados (Stripgenerator, Toondoo, Wittycomics, entre otras).

- Realizar actividades académicas (Content generator, Lim, Webquest creator, LAMS, QuizRevolution, JClic, eduplay, entre otras).

- Marcadores sociales (Digg, Excite, entre otras).

- Entre otros recursos.

\section{III.MeTODOLOGÍA}

La metodología es basada de la investigación basada en diseño (IBD) [17], diseño y exploración de todo tipo de innovaciones educativas, en donde se contemplan también las experiencias relacionadas con implementación de nuevas tecnologías de información y comunicación. Este tipo de investigación es seleccionada ya que posibilita que todas las personas implicadas en el proceso trabajen juntos para mejorar una situación problemática en un contexto real, y por el carácter cíclico en el diseño.

En el proyecto se aplicaron las siguientes técnicas e instrumentos de recolección de información:

- Revisión bibliográfica y de documentación de experiencias

- Observación

- Encuesta

- Experimentación

- Triangulación

Para el proyecto se plantearon las fases de preparación, diseño, implementación, análisis y socialización de resultados.

En las fases de diseño e implementación se utiliza una metodología ágil, la fórmula de ciclos sucesivos conocida como SAM (Successive Aproximation Model) [18], como ilustra la figura siguiente. 


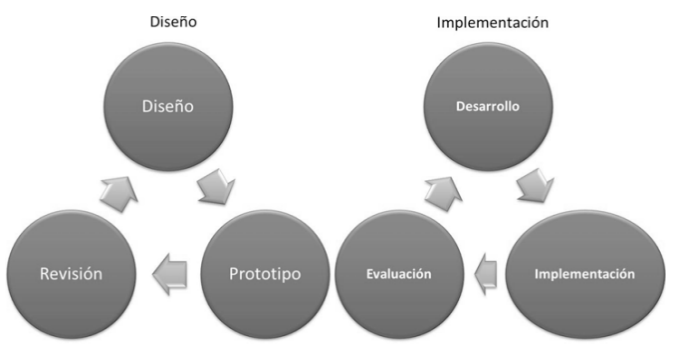

Fig 3. Aplicación de SAM en proyecto. Fuente: Autor

El proyecto se articula con el programa a través del enfoque de la Investigación Acción Participación -IAP- en la que se apunta a la producción de un conocimiento propositivo y transformador, mediante un proceso de debate, reflexión y construcción colectiva de saberes entre los diferentes actores del programa (investigadores, coinvestigadores, estudiantes).

\section{Resultados}

En esta sección se presentarán los resultados de la propuesta de herramienta TIC, un prototipo que actúa como asistente digital para las planeaciones curriculares denominada conTIC, las evaluaciones y posteriormente la propuesta de un diseño de PLE para el docente que incluya a conTIC.

\section{A. Propuesta asistente digital ConTIC}

La herramienta que actúa como asistente para la gestión de unidades de aprendizaje, se denomina conTIC, como ilustra la figura 4.

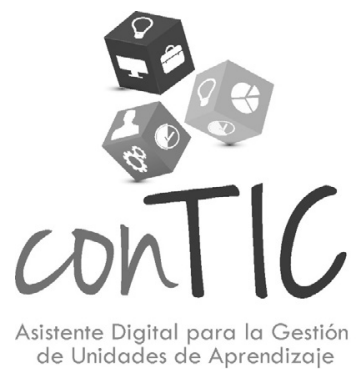

Fig 4. Logo conTIC. Fuente: Autor

Teniendo en cuenta el proceso de planeación del microcurrículo de una asignatura y para el prototipo, se definieron cuatro componentes o etapas principales: caracterización de la planeación, definición de la planeación, ejecución y seguimiento, y finalmente la retroalimentación. En la etapa de caracterización de la planeación, se configura la información de la Institución y de la asignatura que se va a planear. En la etapa de definición de la planeación, se especifican las unidades de aprendizaje, las actividades y los recursos utilizados o necesarios en cada acti- vidad. En la etapa de ejecución y seguimiento, se define las fechas en que la planeación se llevará a cabo, para realizar la autoevaluación de las unidades, recursos y actividades. Conforme se avance, el profesor puede recibir notificaciones y realizar observaciones. Y, en la etapa de retroalimentación, se obtiene el consolidado de la planeación realizada y del seguimiento realizado a la planeación.

El docente con el asistente digital conTIC, figura 5 , podrá a) realizar la planeación de las clases, especificando las unidades de aprendizaje, actividades y recursos, b) obtener recomendaciones de estrategias de enseñanza, actividades y recursos que puede incluir en la planeación, c) obtener ayuda para definir las competencias que se quieren desarrollar en la unidad de aprendizaje y las estrategias que permiten hacerlo, d) interactuar con los docentes que participan. Además con el asistente digital conTIC, el docente puede hacer seguimiento y retroalimentación del proceso.

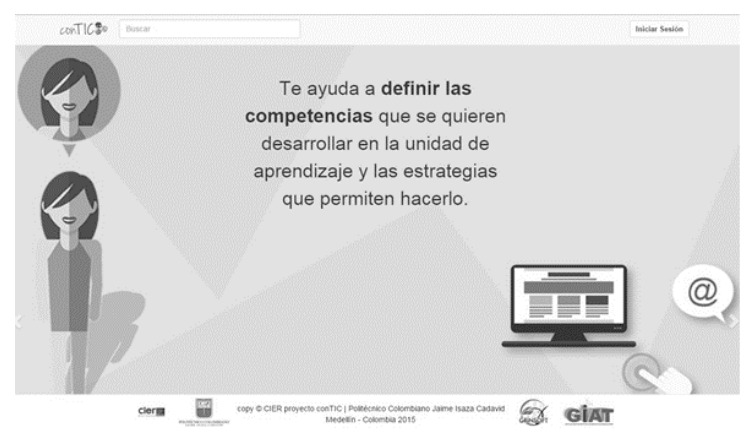

Fig. 5. Ejemplo interfaz inicial conTIC. Fuente: Autor http://54.174.104.82:3000/

\section{B. Evaluación del prototipo conTIC}

Para efectos de evaluar el prototipo y proyectar las mejoras, se definieron tres fases, como ilustra la figura 6. Primero se diseñaron los instrumentos para la encuesta (http://goo.gl/forms/yHrZVxIXrF), seguidamente junto con la prueba del aplicativo se aplica la encuesta a dos docentes en cada una de las instituciones educativas (Gabriel García Márquez, e INEM José Félix de Restrepo), y posteriormente se obtiene los resultados.

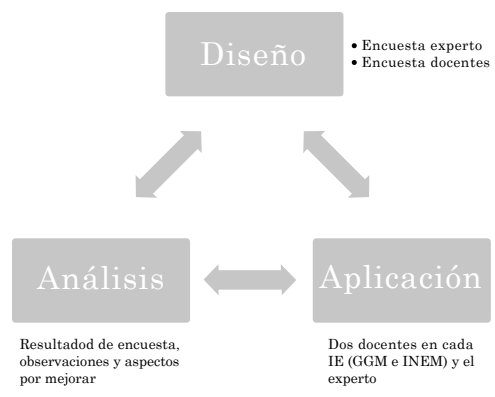

Fig. 6. Proceso evaluación conTIC. Fuente: Autor 
La encuesta contempló aspectos pedagógicos como técnicos. Algunos de los resultados se indican a continuación. Para un $100 \%$ de los encuestados estaban de acuerdo con la satisfacción de conTIC como asistente, según las necesidades y expectativas, ver figura siguiente.

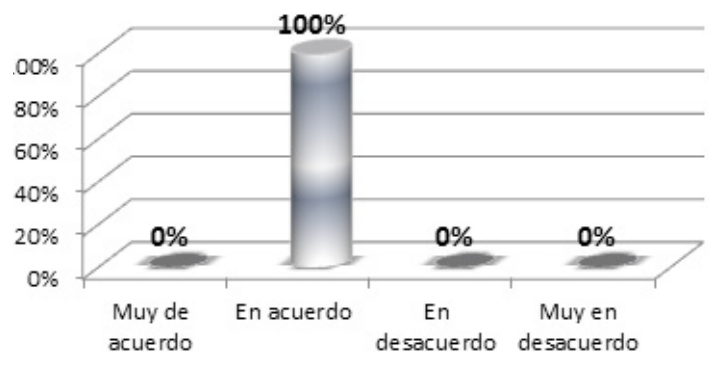

Fig. 7. Resultado satisfacción conTIC. Fuente: Autor

Para un $75 \%$, estaban de acuerdo en que ConTIC contempla elementos de la planeación particular del docente, ver figura 8.

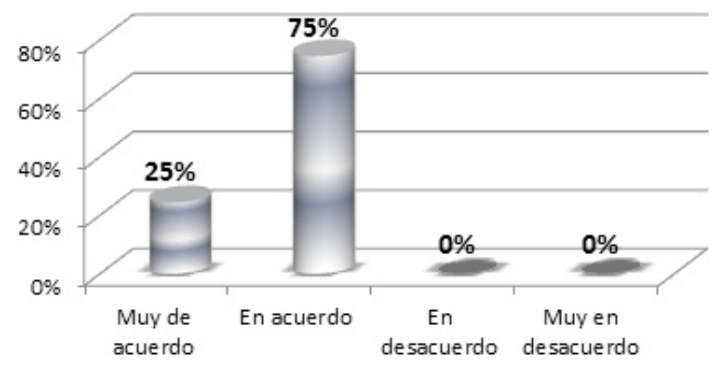

Fig. 8. Resultado elementos planeación conTIC. Fuente: Autor

Por otro lado frente a la pregunta de sí el uso de ConTIC es intuitivo, un $75 \%$ estaban de acuerdo, y $25 \%$ en desacuerdo, ver figura 9. Por lo cual habría que trabajar en mejorar este aspecto.

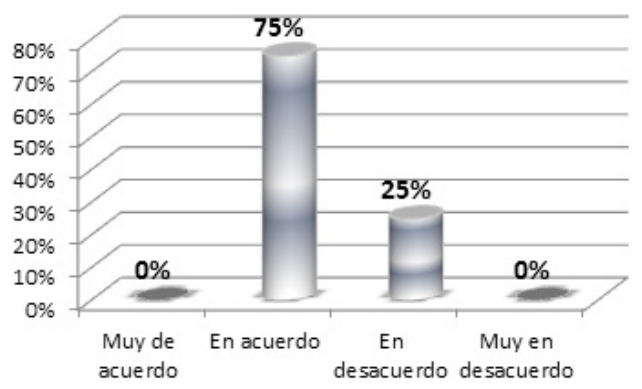

Fig. 9. Resultado uso-intuitivo. Fuente: Autor

Para el $75 \%$ estaban en desacuerdo en que el asistente presenta canales de comunicación para interactuar con otros docentes, y un $25 \%$ en muy desacuerdo, ver figura 10. Si bien el prototipo contempló un chat para la comunidad, se requiere mejorar significativamente este aspecto.

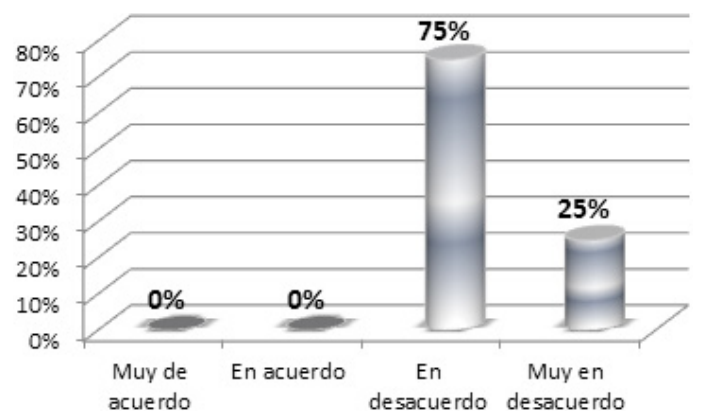

Fig. 10. Resultado comunicación-interacción. Fuente: Autor

De acuerdo al análisis general de los resultados del prototipo conTIC, se tiene una buena aceptación de la herramienta como tal para ayudar a planear las clases, contempla elementos pedagógicos para definir las unidades de aprendizaje, competencias, actividades y recursos, pero habrá que mejorar ciertos aspectos técnicos del aplicativo web. Entre los aspectos a mejorar es la necesidad de implementar en el asistente diversos canales de comunicación que permitan la interacción con docentes de la misma Institución y de otras, facilitando la conformación de comunidades de aprendizaje según el área de formación, práctica o intereses personales. Entre otros aspectos a mejorar se tienen:

- Que permita trabajar de forma colaborativa en la planeación.

- La navegabilidad e intuición al usar la herramienta.

- Tener conexión con varios repositorios de contenidos educativos.

- Comunicación y seguimiento a estudiantes

- La consistencia en dar uno o dos clics en los botones.

- Añadir componentes de accesibilidad

- Plantilla configurable por el docente.

- Recibir las notificaciones vía correo.

- La opción de ayuda sea más óptima.

- Contemplar otros aspectos como (pregunta problematizadora, logros, indicadores de desempeño).

\section{Propuesta diseño PLE docente incluyendo a conTIC}

A la hora de diseñar un PLE para el docente, se deben considerar muchos aspectos, entre ellos los personales (gustos, intereses, experiencias, conocimientos, entre otros), identificar las fuentes de información, las herramientas para crear información, y con cuales se comparte la información. Por otro lado es una labor de explorar y aprender, a usar y apropiarse de las TIC. Esto puede ser a definición y elección de cada individuo, por lo cual en la propuesta que se plantea, se hace referencia a varias herramientas (que bien pueden ser otras), y a la incorporación de la herramienta digital conTIC para 
apoyar en la planeación de las clases, abriendo más el abanico que conforma el PLE del docente. En la figura 11, se presenta la propuesta de un PLE para el docente.

Se resaltan cuatro partes resaltadas por Moreno [19] que contemplan un PLE:

Fuente de información: Es donde el usuario, en este caso el docente obtiene la información, y como "aprende". Desde el contexto en que se mueve, su institución educativa, Ministerio de Educación, hasta las herramientas como buscador de google, flickr, youtube, pinterest, scoop it, RSS, correos en google o yahoo, redes sociales como twitter, páginas web específicas, cursos en Moodle, Udacity, repositorios de objetos de aprendizaje, información proveniente de publicaciones arbitradas (ej, IEEE, Science direct, etc) o bases de datos o libros reconocidos. En el caso en se use el asistente digital conTIC, desde esta etapa hay información que le será útil al docente para planear, definir actividades y recursos.

Organizar-seleccionar: Dada la posible cantidad de información, el usuario o docente debe filtrar, eliminar, organizar y seleccionar la información que es primordial o de importancia. Puede usar herrramientas como Scoop it, Pinterest, dropbox, google drive, delicious, "basurero", zotero, evernote, entre otras. En el caso en que se use el asistente digital conTIC, en esta etapa se prepara y decide la información o recursos a usar (ej, links a determinado recurso).

Crear: Es donde el usuario o docente crea contenidos/información relacionados al proceso de enseñanza/aprendizaje y/o de investigación o profesional. Desde cualquier sistema operativo en que trabaje el docente (Windows, iOS, android, Linux) puede usar diversas herramientas para editar textos, imágenes, videos, presentaciones, plataformas como Joomla, entre otros, y el asistente digital para gestionar unidades de aprendizaje conTIC. En el caso de usar conTIC, se crea las planeaciones.

Comunicar - compartir: Es donde el usuario o docente propicia que los contenidos/información creados, sean publicados y compartidos al público de interés. Herramientas como Blog, site, Moodle, google + , dropbox o google drive, youtube, redes sociales twitter o facebook, whatsApp, correos electrónicos, la publicación en revistas preferiblemente indexadas y ponencias en eventos, entre otros pueden usarse. Para el caso en que se use el asistente conTIC, el usuario puede interactuar por medio de un chat y compartir sus planeaciones con otros docentes.

\section{Conclusiones}

Se presentó un resumen de plataformas y herramientas educativas, y una propuesta del asistente conTIC como otro recurso digital del entorno de aprendizaje personal- PLE para el docente, brindándole la posibilidad al docente que lo elija y se apropie de la herramienta, como asistente para la planeación de las clases.

Los PLE contempla tanto aspectos pedagógicos y tecnológicos. Cada docente define según su experiencia, necesidades, e intereses los diferentes recursos TIC para sus labores. Así, el prototipo conTIC, se propone como otra herramienta TIC para apoyar la gestión de unidades de aprendiza-

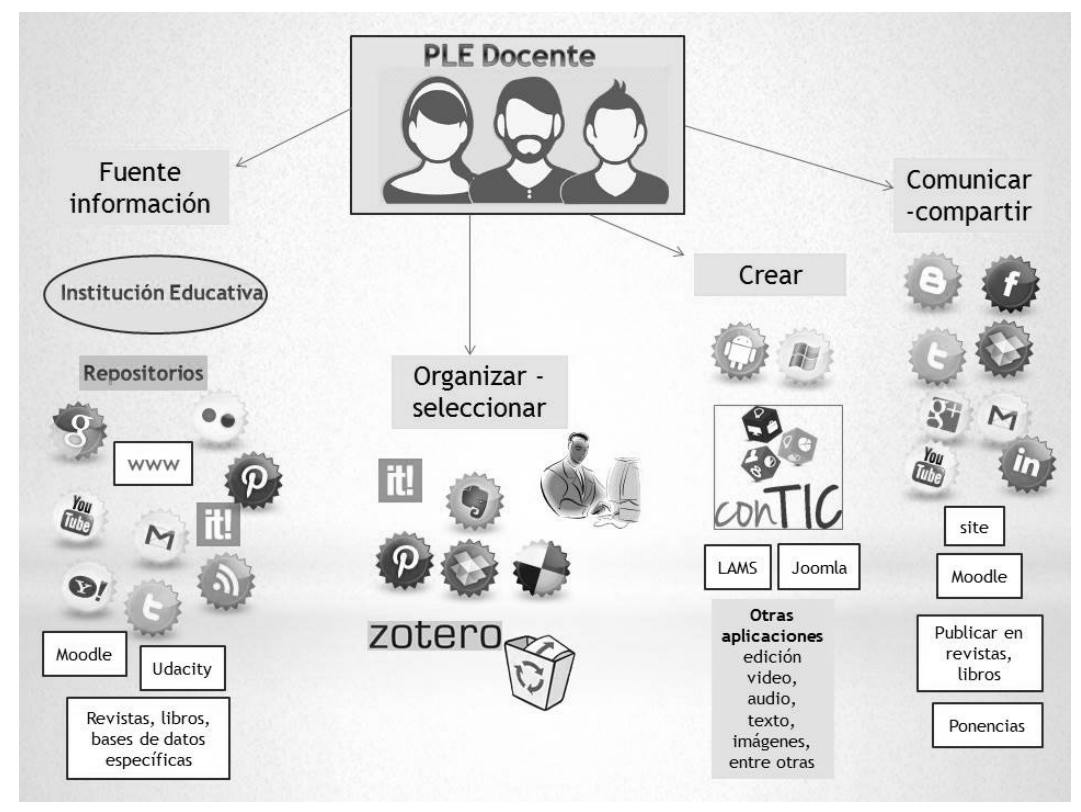

Fig. 11. Propuesta PLE docente con asistente conTIC.

Fuente: Autor 


\section{PLATAFORMAS Y HERRAMIENTAS EDUCATIVAS COMO PARTE DEL PLE DEL DOCENTE. CASO ASISTENTE DIGITAL PARA PLANEACIÓN CURRICULAR CONTIC}

je. Sumado a que el docente use significativamente las TIC, y se planeen mejor las clases, habrá mayor calidad en el proceso de enseñanza y aprendizaje. Dada las ventajas de las TIC, la apropiación de estas al proceso educativo es un ejercicio continuo.

El factor diferenciador del asistente digital propuesto, es que es basado de los estándares de conocimiento y las opciones de acceder a los repositorios de objetos de aprendizaje definidos por el Ministerio de Educación Nacional. El asistente sirve para cualquier área de conocimiento o asignaturas de propósito general, y en principio en el contexto Colombiano y de carácter libre con todas las características.

El prototipo como tal fue probado y validado, realizando chequeo de su funcionalidad, y verificando su propósito para gestionar unidades de aprendizaje. En general los resultados de la encuesta fueron satisfactorios, resaltando aspectos técnicos y pedagógicos, lo cual indica que sí podría tener aceptación y opciones de implementación a gran escala, y proyectando las mejoras. Contic, como prototipo es un buen insumo para posterior trabajo de depuración y puesta a punto.

De acuerdo a las pruebas y resultados, los encuestados manifiestan según sus expectativas y necesidades, estar de acuerdo con un aplicativo web que apoye el proceso de planeación de las clases, indicando satisfacción con la propuesta del prototipo conTIC. Resaltan los elementos de la planeación y que se pueda definir la competencia, las actividades, y recursos asociados.

Como temáticas de investigación y desarrollo a futuro, se proyectan: depuración del prototipo e implementación masiva en diversas Instituciones educativas; Análisis y diseño para despliegue en diferentes plataformas (nube, servidores locales, app en android e ios, entre otros), articulación con diferentes repositorios de acceso libre indicados en Opendoar (Directory of Open Access Repositories) y en ROAR (Registry of Open Access Repositories); articulación con otras plataformas educativas como LMS, CMS, LCMS, LAMS; Involucrar más desarrollo de inteligencia artificial, para que la herramienta pueda aprender, inferir y sea cada vez más un "asistente" como tal, para recomendar, buscar, filtrar, comunicar, entre otros; Implementación y análisis dentro de un escenario de PLE que involucre diferentes recursos y estrategias.

\section{REFERENCIAS}

[1] R. Carneiro, J. C. Toscano, and T. Díaz, Los desafíos de las TIC para el cambio educativo. Metas Educativas 2021. Organización de Estados Iberoamericanos para la Educación, la Ciencia y la Cultura (OEI). España: Fundación Santillana, 2009.

[2] Magisterio, "Programa Todos a Aprender, PTA," 08Nov-2014. .

[3] A. Pampillón, "Las plataformas e-learning para la enseñanza y el aprendizaje universitario en Internet." Universidad Complutense de Madrid, 2009.

[4] L. Castañeda and J. Adell, Entornos Personales de Aprendizaje: claves para el ecosistema educativo en red. Alcoy: Marfil, 2013.

[5] G. Siemens, "Connectivism: A learning theory for the digital age," International Journal of Instructional Technology and Distance Learning, vol. 2, no. 1, pp. 3-10, Jan-2005.

[6] M. Llamas-Nistal, M. Caeiro-Rodriguez, J. Gonz'lezTato, and J. Alvarez-Osuna, "Integrating Web Services with Gadgets to Support an i-Google PLE," 2012, pp. 381-382.

[7] C. K. N. C. K. Mohd and F. Shahbodin, "Personalized Learning Environment (PLE): Need Analysis in Malaysian's Secondary School," in e-Learning, e-Management and e-Services (IC3e), 2013 IEEE Conference on, Sarawak, 2013, pp. 79-82.

[8] L. Moccozet, O. Benkacem, H. Platteaux, and M. Foerster, "Looking for a Platform That Can Be Proposed to Students as a PLE Enabler," 2014, pp. 498-503.

[9] Universidade de Aveiro, "Sapo Campus na UA," Sapo Campus na UA.

[10] Ministerio de Ciencia e Innovación del Gobierno de España, "Proyecto Dipro," 2009. .

[11] Eduteka and FGPU, "CURRÍCULO INTERACTIVO 2.0 PARA EL ÁREA DE INFORMÁTICA,” Modelo Curricular Interactivo, 01-Jun-2008. .

[12] S. M. Castro, C. A. Clarenc, C. López de Lenz, M. E. Moreno, and N. B. Tosco, ANALIZAMOS 19 PLATAFORMAS DE E-LEARNING, 2013th ed. 2013.

[13] S. Ninoriya, P. M. Chawan, B. B. Meshram, and M. VJTI, "CMS, LMS and LCMS For eLearning," IJCSI Int. J. Comput. Sci. Issues, vol. 8, no. 2, pp. 644-647, 2011.

[14] Macquarie University, "LAMS," LAMS Foundation, 2015-2002.

[15] Open Universiteit Nederland, "CopperCore," Nov-2008. .

[16] Relpe, "Herramientas Educativas," Red Latinoamericana portales educativos, 23-Feb-2015. .

[17] T. Gibelli, La investigación basada en diseño para el estudio de una innovación en educación superior que promueve la autorregulación del aprendizaje utilizando TIC. Madrid: FIECC, 2009.

[18] Allen Interactions Inc, "Agile eLearning Development with SAM | Allen Interactions," 2015. [Online]. Available: http://www.alleninteractions.com/sam-process. [Accessed: 13-Dec-2015].

[19] G. A. Moreno López, "Curación de contenido y panel de recuperación,” Aprendizaje everywhere, 30-Jun-2015. . 